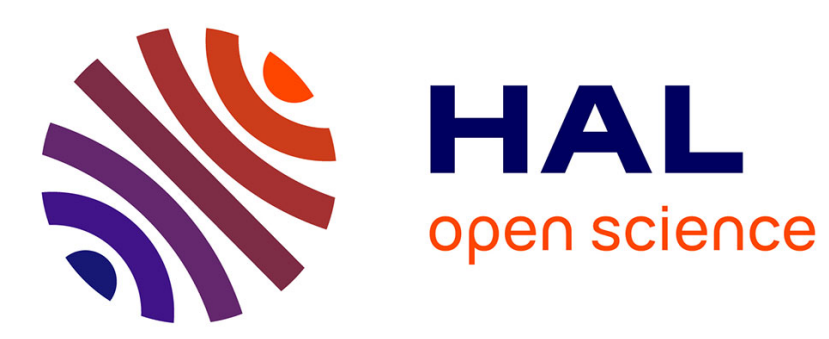

\title{
The free-energy landscape of single-molecule polymer crystals
}

\author{
Luca Larini, Dino Leporini
}

\section{To cite this version:}

Luca Larini, Dino Leporini. The free-energy landscape of single-molecule polymer crystals. Philosophical Magazine, 2007, 87 (3-5), pp.411-415. 10.1080/14786430600860993 . hal-00513731

\section{HAL Id: hal-00513731 \\ https://hal.science/hal-00513731}

Submitted on 1 Sep 2010

HAL is a multi-disciplinary open access archive for the deposit and dissemination of scientific research documents, whether they are published or not. The documents may come from teaching and research institutions in France or abroad, or from public or private research centers.
L'archive ouverte pluridisciplinaire HAL, est destinée au dépôt et à la diffusion de documents scientifiques de niveau recherche, publiés ou non, émanant des établissements d'enseignement et de recherche français ou étrangers, des laboratoires publics ou privés. 


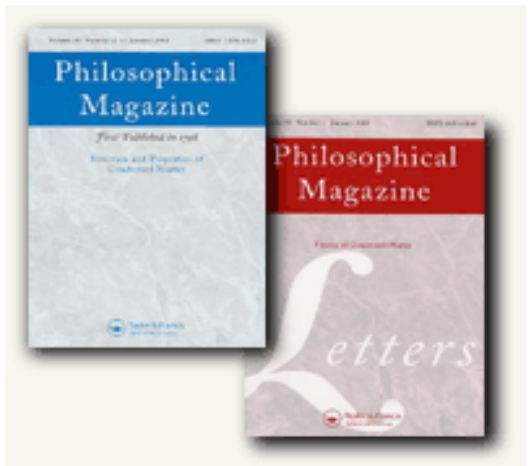

\section{The free-energy landscape of single-molecule polymer crystals}

\begin{tabular}{|r|l|}
\hline Journal: & Philosophical Magazine \& Philosophical Magazine Letters \\
\hline Manuscript ID: & TPHM-06-Apr-0114.R1 \\
\hline Journal Selection: & Philosophical Magazine \\
\hline $\begin{array}{r}\text { Date Submitted by the } \\
\text { Author: }\end{array}$ & 12-Jun-2006 \\
\hline Complete List of Authors: & $\begin{array}{l}\text { Larini, Luca; University of Pisa, Physics } \\
\text { Leporini, Dino; University of Pisa }\end{array}$ \\
\hline Keywords: & molecular dynamic simulations, polymers \\
\hline \multicolumn{2}{|c|}{} \\
\hline $\begin{array}{l}\text { Note: The following files were submitted by the author for peer review, but cannot be converted } \\
\text { to PDF. You must view these files (e.g. movies) online. }\end{array}$ & polymer crystals, free-energy landscape \\
\hline MolvenoCryst_rev.tex \\
\hline
\end{tabular}

\section{(5) ScholaroNE \\ Manuscript Central}




\title{
The free-energy landscape of single-molecule polymer crystals
}

\author{
L. LARINI, D. LEPORINI* \\ Dipartimento di Fisica "Enrico Fermi", Università di Pisa, Largo B. \\ Pontecorvo 3, I-56127 Pisa, Italy \\ and \\ INFM-CRS SOFT, Largo B.Pontecorvo 3, I-56127 Pisa, Italy \\ ( Received: June 12, 2006 )
}

By using extensive MD simulations the free-energy landscape of the crystallization process of a single polyethylene chain with $N=500$ monomers is determined and the roles of both the potential energy and the entropy to shape its gross features are discussed.

Keywords: Polymers; Single-molecule crystals; Molecular-dynamics simulations

\section{Introduction}

Folded states of chain-like macromolecules like crystalline polymers are under current intense study. One key issue is if their morphologies are thermodynamically or kinetically controlled. Kinetic factors are believed to set the growth rate of polymer crystals as well as the thickening of folded macromolecules $[1,2]$. While this is a safe conclusion for long chains ( polymers ), where large entropic barriers hamper the conformation changes leading to structures which are e.g. partially crystalline, it may be questioned for shorter chains ( oligomers ) which are less impeded. The crystalline state of polymers is very different from that of other materials because of the need to arrange in an ordered way a large number of monomers linked to each other sequentially. This results in a wide range of possible hierarchical morphologies where the basic unit is the lamella, which is a few hundred Angstrom thick [1,2]. The backbone of a single polymer chain, which is several thousand Angstrom long, is folded inside the lamella to form the so-called stems; these are perpendicular to the basal surfaces of the lamella where the loops connecting the stems are localized [2]. Recently, numerical simulations showed that single-chain polymer crystals are equilibrated, i.e. the state corresponds to the minimum of the free-energy and is not kinetically

*Author for correspondance. E-mail: dino.leporini@df.unipi.it 
selected $[3,4,5,6,7,8]$. The present paper reports on a study, carried out by Molecular-Dynamics ( MD ) algorithms, of the energy and the entropy contributions to the free-energy of single-chain crystals of polyethylene ( $\mathrm{PE}$ ) .

\section{$2 \quad$ Numerical methods}

The behaviour of a single $\mathrm{PE}$ chain with $N=500$ monomers in solution has been studied by means of a united-atom model $[3,4]$. The solvent is mimicked by suitable friction and random forces acting on the monomers. The local interactions set the bond length distance, the bond angle, and the torsional barrier defined for every quadruplet of adjacent beads. Fig. 1 plots the dihedral torsional energy. Pair of beads not interacting by any of the preceding potentials interact via a Lennard-Jones ( LJ) potential. The dynamics is described by a Langevin equation which is integrated by means of the velocity Verlet algorithm with time step $\Delta t=10^{-3}$. Further details are given elsewhere [3]. The time and temperature units are given by $t^{*}=2.21$ ps and $T^{*}=56.3 \mathrm{~K}$. All the results are presented in terms of reduced units. Following the initial equilibration at $T_{e q}=15$, the final temperature $T_{f}=9$ is reached via different thermal histories to test possible memory effects [3]. Afterwards, the crystallization process develops under isothermal conditions. Several runs have been performed to ensure proper statistics.

\section{Results and Discussion}

The kinetics of the crystallization process depends on the chain conformation. The nucleation may involve either the whole chain or separate portions merging afterwards by reeling in their connector $[3,8]$. Our interest is in the collective rearrangements occurring after that preliminary stage. At later times, following different paths along which metastable intermediate crystalline structures with different number of stems are assembled and restructured, the molecule comes to a final crystalline structure, where the chain is folded with ten stems which is independent of the thermal history [3]. The crystal presents a rod-like shape, being characterized by the three moments of inertia $I_{i}, i=1,2,3$ with $I_{1} \gtrsim I_{2} \gg I_{3}$.

The structural changes of the crystalline structures are twofold: i) changes due to the increase or decrease of the number of stems [3]; ii) changes which leave the number of stems unaltered $[3,5]$. The former are effectively characterized by the moment of inertia $I_{1}$ and are activated. The latter do not lead to either appreciable changes of $I_{1}$ or the internal energy. Examples of such processes are provided by the reorganizations taking place on the crystal surface, e.g. two stems are seen to slide on the crystal surface while preserving their alignment with the ordered substrate [5]. Another kind of restructuring with no change of the number of stems is the position exchange between aligned stems, e.g. the expulsion of a stem terminated by one chain-end from

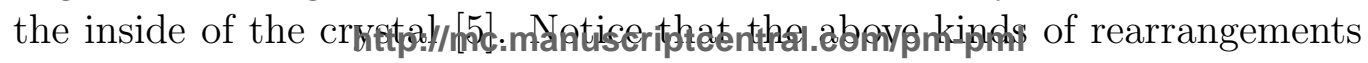


leave the potential energy largely unaffected, i.e. they are entropic processes, with negligible changes of the moments of inertia.

In order to characterize the reorganization of the chain, both the free energy $F$ and the potential energy $U$ are sampled and their average over all the available configurations correlated to the order parameter $I_{1}$. The free energy is evaluated according to the Landau's formula $F(X)=-k_{B} T \ln P(X)$ where $P(X)$ is the probability to find the macroscopic order parameter with value $X[9]$. In the present case the choice $X=I_{1}$ is made in agreement with others $[7,8]$. In fact, the crystalline structures are rodlike with foldings having, approximately, equal length [3]. Consequently, the metastable states are well identified by the length of their stems $m$ and then by $I_{1}$, the latter being roughly proportional to $m^{2}$. Efficient Monte Carlo simulations may be also used to evaluate the free energy. However, MD techniques, which were already extensively used to investigate the crystallization dynamics of the present system $[3,4,5,6]$, proved to be accurate enough to the present purposes.

Figure 2 plots the free-energy landscape ( FEL) at $T=9$, the overall potential energy and its torsional and LJ contributions as functions of the largest moment of inertia of the chain $I_{1}$. For a given $I_{1}$ value the above quantities are the averages over all the configurations with equal $I_{1}$ assumed by the crystal during the time evolution. Since bond angles and length exhibit negligible changes, the related energies are not considered. The range of $I_{1}$ values and the FEL statistics in Fig.2 is much improved with respect to previous reports $[3,5]$, thus allowing for a more detailed view of the metastable structures. Several minima of the free energy, corresponding to well-defined metastable crystals with different number of stems, are seen. The global minimum corresponds to the equilibrated lamella with ten stems. Figure 2 shows that the free-energy exhibits minima close to the potential energy ones. The discussion on both the free-energy barriers and the energy ones was presented elsewhere [5]. Here, we are interested in the gross features of both the FEL and the average potential energy landscape. First, we focus on the potential energy with contributions from both the torsional and the LJ potentials. The decrease of the torsional energy by increasing $I_{1}$, i.e. by decreasing the number of stems, which is seen in Fig. 2 is due to the conversion of $g_{ \pm}$torsional states into $t$ states ( see fig.1) leading to longer stems. On the other hand, increasing $I_{1}$ leads to more prolate crystals where the attractive tail of the LJ potential becomes more important. This explains the increase of the LJ energy by increasing $I_{1}$ which is seen in Fig. 2. The different $I_{1}$ dependences of the torsional and LJ potential energies set the overall curvature of the total potential energy. Fig. 2 also shows that the latter has the global minimum when the crystal has seven stems. This differs from the FEL global minimum being found for crystals with ten stems. The effect is due to entropic effects shaping the gross features of the FEL. It was shown elsewhere that the combinatorics of the stems and of the loops provides a convenient model [4] . 


\section{Conclusions}

MD simulations of the crystallization of a single polyethylene chain shows that the folding process involves intermediate metastable crystalline states and ends up in an equilibrated lamella with ten stems. The roles of both the potential energy and the entropy to shape the gross features of the FEL were discussed.

\section{References}

[1] G. Strobl, The Physics of Polymers (Springer, New York, 1997).

[2] K. Armitstead, G. Goldbeck-Wood, Adv. Polym. Sci. 100219 (1992).

[3] L.Larini, D.Leporini, J. Chem. Phys. 123144907 (2005).

[4] L. Larini, A. Barbieri, D. Prevosto, P.A. Rolla, D. Leporini, J. Phys.: Condens. Matter 17 L199 (2005).

[5] L. Larini, D. Leporini, J. Non-Crystalline Sol. in press.

[6] L. Larini, A.Barbieri, D. Leporini, Physica A 364183 (2006).

[7] P. Welch, M. Muthukumar, Phys. Rev. Lett. 87218302 (2001).

[8] M. Muthukumar, Phil.Trans. R.Soc. Lond. A 361539 (2003).

[9] W.Hu , D.Frenkel, V.B.F.Mathot, J. Chem. Phys. 1183455 (2003). 


\section{Figure captions}

Figure 1: The dihedral torsional energy as function of the dihedral torsional angle in units of $k_{B} T$ with $T=9$ in reduced units. $\Delta U$ is the energy change in a $g_{ \pm} \leftrightarrow t$ transition.

Figure 2: From the top to the bottom: the free-energy landscape ( FEL ) at $T=9$, the overall potential energy and its torsional and LennardJones contributions as function of the largest moment of inertia of the chain $I_{1}$. The labels indicate the number of stems $\mu$ of the metastable crystals corresponding to the free-energy minima. 


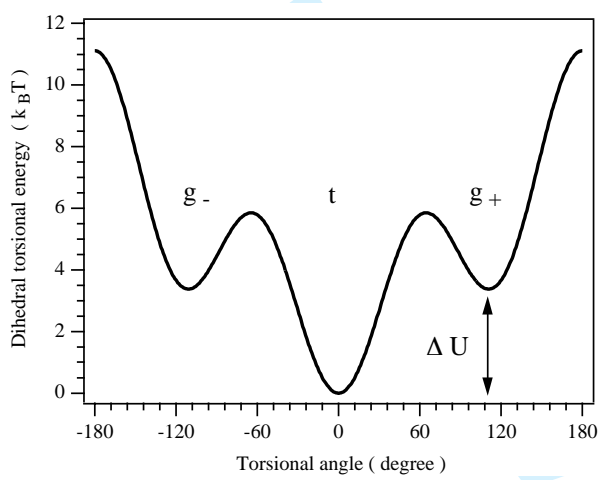

FIGURE 1 


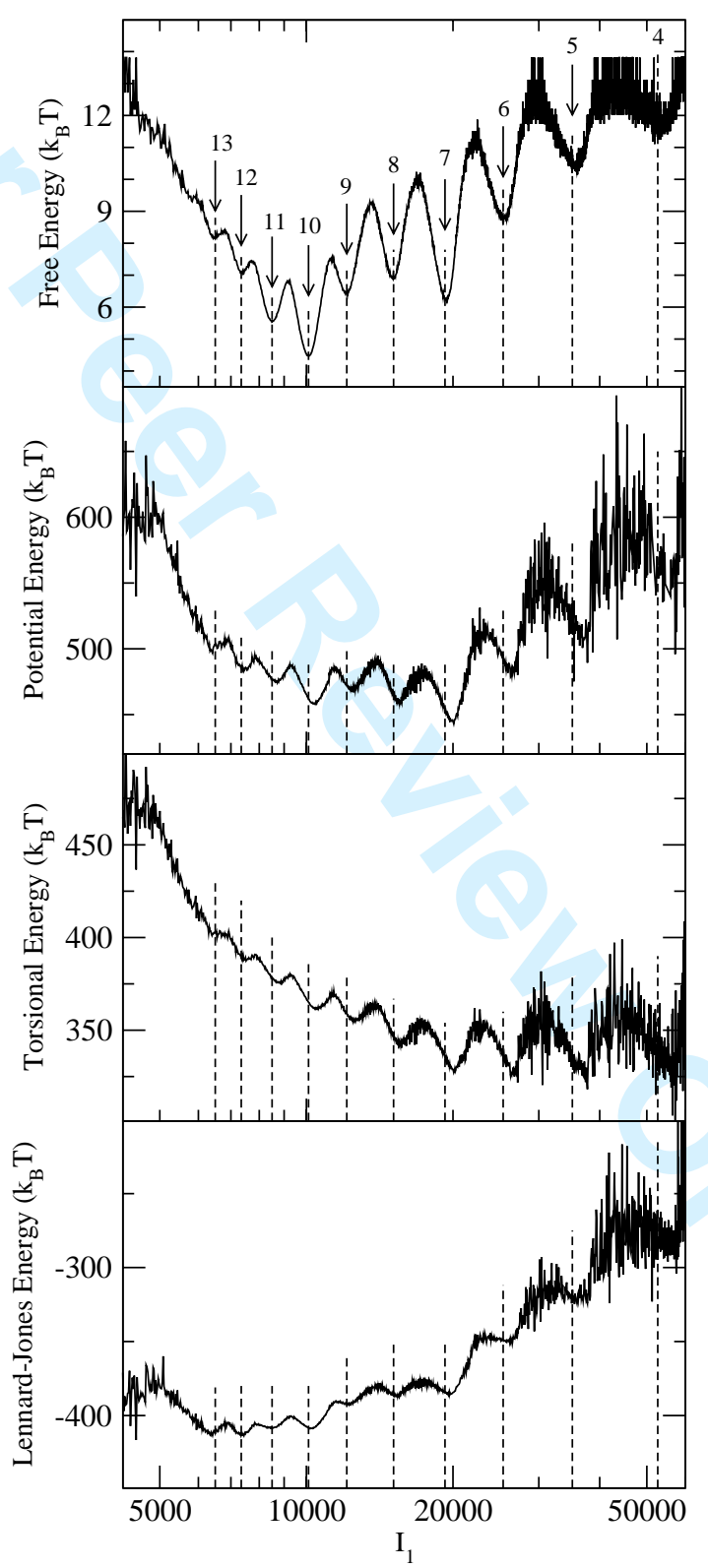

FIGURE 2 
